\section{Psoriasis-Arthritis: Karotis-Ultraschall- Untersuchung detektiert Gefäßschäden}

Martínez-Vidal MP et al. Role of Carotid Ultrasound and Systematic Coronary Risk Evaluation Charts for the Cardiovascular Risk Stratification of Patients With Psoriatic Arthritis. J Rheumatol 2020; 47: 682-689

Im Rahmen einer multizentrischen Studie wurde das kardiovaskuläre Risiko (CV) von Patienten mit Psoriasis-Arthritis (PsA) bewertet, das Vorhandensein einer subklinischen Herz-Kreislauf-Erkrankung mittels standardisierter Karotis-Ultraschall-Untersuchung bestimmt und der mögliche Zusammenhang zwischen CV-Erkrankung und PsA-Merkmalen ermittelt.

In die deskriptive Querschnittsstudie, die im Südosten von Spanien durchgeführt wurde, wurden Patientinnen und Patienten über 18 Jahre eingeschlossen, die die CASPAR-Klassifikationskriterien für Psoriasis-Arthritis erfüllten. Das klinische CV-Risiko über eine 10-Jahres-Periode wurde mit SCORE-Diagrammen (Systematic Coronary Risk Evaluation) berechnet. Ultraschalluntersuchungen der Halsschlagader erfolgten, um die Intima-Media-Dicke der Karotiswand und das Vorhandensein von Atherom-Plaques zu bewerten. Die Patientinnen und Patienten wurden nach Ultraschall-Ergebnissen neu eingestuft. Um Assoziatio- nen von Ultraschall-Karotisanomalien mit klassischen CV-Risikofaktoren und PsAMerkmalen zu testen, kam als statistisches Verfahren die multivariate Regression zum Zuge.

Insgesamt wurden 176 Patienten Patientinnen und Patienten mit PsA, 87 Frauen und 89 Männer, in die Studie eingeschlossen. Das Durchschnittsalter lag bei 55,2 $\pm 11,8$ Jahren. Nahezu alle hatten eine Psoriasis (95\%).

Das CV-Risiko aller PsA-Patientinnen und Patienten gemäß SCORE war gering. Bei 65,3\% der Studienteilnehmer war das SCORE-geschätzte CV-Risiko mittelschwer (SCORE 1-4), 12,5\% hatten ein hohes (SCORE5-9) und 1,1\% ein sehr hohes Risiko (SCORE $\geq 10$ ). Insgesamt 27 Patientinnen und Patienten hatten einen Diabetes mellitus und 4 von Ihnen bereits CV-Ereignisse in der Vergangenheit. Diese Personen gehörten alle zu den Gruppen mit hohem und sehr hohem Risiko.

In der Ultraschall-Untersuchungen fanden sich bei 26,3\% der Patientinnen und Patienten Atherome (gemäß Mannheim IntimaMedia Thickness Consensus) und bei weiteren 10 Patientinnen und Patienten eine erhöhte CIMT >0,9 mm.

Insgesamt wurden 53 Patientinnen und $\mathrm{Pa}$ tienten (30,8\%) aufgrund der UltraschallBefunde in die Gruppe mit sehr hohem Risiko umgestuft.

In der multivariaten Regressionsanalyse ergab sich eine Assoziation mit dem Vorhandensein von Plaques nur mit den Variablen Alter und Dyslipidämie, jedoch nicht mit anderen Risikofaktoren.

Bei Patienten mit mittlerem Risiko war eine axiale Erkrankung mit Karotisanomalien assoziiert. Axiale Erkrankung und höhere Harnsäurewerte waren mit einer Einstufung in höheren Risikogruppen assoziiert. Bei Patienten mit klinisch hohem Risiko führten höhere C-reaktiven Proteinspiegel häufiger zu einer Höhergruppierung. 
FAZIT

Viele Patientinnen und Patienten mit PsA haben ein klinisch geschätztes mittleres oder hohes CV-Risiko. Eine Ultraschall-Untersuchung der Karotis detektiert subklinische Gefäßerkrankungen und ist durchaus nützlich, zur Einschätzung des tatsächlichen Risikos. Denn die Existenz von Atheromen kann nur teilweise durch die klassischen CV-Risikofaktoren erklärt werden, schreiben die Autorinnen und Autoren der Studie.

Richard Kessing, Zeiskam 